VOLUME 30 (2021) 243-259

DOI: $10.24330 /$ ieja.969917

\title{
C-CANONICAL MODULES
}

Mohammad Bagheri and Abdol-Javad Taherizadeh

Received: 8 August 2020; Revised: 13 January 2021; Accepted: 25 January 2021

Communicated by A. Çiğdem Özcan

\begin{abstract}
Let $C$ be a semidualizing module over a commutative Noetherian local ring $R$. In this paper we introduce a new class of modules, namely $C$-canonical modules which are a generalization of canonical modules. It is shown that if the canonical module exists then the $C$-canonical module exists and the converse holds under special conditions. Also, a new characterization of Gorenstein local rings is given via $C$-canonical modules.
\end{abstract}

Mathematics Subject Classification (2020): 13C05, 13D05, 13D07, $13 \mathrm{H} 10$

Keywords: Semidualizing modules, dualizing modules, local cohomology, Auslander class, Bass class

\section{Introduction}

Throughout this introduction $(R, \mathfrak{m})$ is a commutative Noetherian local ring of dimension $n, \mathrm{E}_{R}(R / \mathfrak{m})$ denotes the injective envelope of $R / \mathfrak{m}$ and $\mathrm{H}_{\mathfrak{m}}^{n}(R)$ is the $n$-th local cohomology module of $M$ with respect to $\mathfrak{m}$.

Grothendieck [11] defined a canonical module over a complete local ring and called it a module of dualizing differentials; see [11, page 94]. Herzog and Kunz defined a canonical module for $R$ as a finitely generated $R$-module $K$ for which $K \otimes_{R} \hat{R} \cong \operatorname{Hom}_{R}\left(\mathrm{H}_{\mathfrak{m}}^{n}(R), \mathrm{E}_{R}(R / \mathfrak{m})\right)$ [12, Definition 5.6]. In [13] M. Hochster and C. Huneke, defined a canonical module as a finitely generated $R$-module $K$, for which $\operatorname{Hom}_{R}\left(K, \mathrm{E}_{R}(R / \mathfrak{m})\right) \cong \mathrm{H}_{\mathfrak{m}}^{n}(R)$. By a dualizing module over a Cohen-Macaulay local ring, we mean a finitely generated maximal Cohen-Macaulay $R$-module with finite injective dimension of type 1 (see Section 2 for the definition of type). A canonical module of a Cohen-Macaulay local ring (if it exists) actually is the dualizing module. Canonical modules play an important role in studying Cohen-Macaulay local rings.

It is known that a canonical module (if it exists) is unique up to isomorphism [3, Theorem 12.1.6]. Canonical modules in general are studied extensively in the literature. Aoyoma [2] proved excellent results concerning behavior of canonical modules under flat base change, the endomorphism ring of canonical modules, and 
the trivial extension of the ring by canonical modules. In [1], the author proved that if the canonical module has finite projective dimension, then it is isomorphic to $R$. Also, it is known that if $R$ is a homomorphic image of a Gorenstein local ring, then it has the canonical module, and the converse holds when $R$ is Cohen-Macaulay. In fact, when $R$ is a Cohen-Macaulay local ring, Foxby [8], and Reiten [16] proved (independently) that if the canonical module exists, then the trivial extension of $R$ by the canonical module is a Gorenstein local ring, and thus $R$ is a homomorphic image of a Gorenstein local ring.

A semidualizing $R$-module is a finitely generated $R$-module $C$ such that the homothety map $R \longrightarrow \operatorname{Hom}_{R}(C, C)$ is an isomorphism, and $\operatorname{Ext}_{R}^{i}(C, C)=0$ for all $i>0$. These modules, were introduced by Foxby [8], Vasconcelos [19], and Golod [10] independently. The ring itself and the dualizing module (if it exists) are examples of semidualizing modules. Semidualizing modules have been studied by many researchers; see, for example, [5], [6], [9], [15], [18], [20]. Also, we refer the reader to [21] for detailed results concerning semidualizing modules.

The main goal of this paper is to generalize the concept of canonical modules for semidualizing modules. To do this, we define a $C$-canonical module (or a canonical module for $C)$ as a finitely generated $R$-module $K$ such that $\operatorname{Hom}_{R}\left(K, \mathrm{E}_{R}(R / \mathfrak{m})\right) \cong$ $\mathrm{H}_{\mathfrak{m}}^{n}(C)$, where $C$ is a semidualizing module.

In Section 3, we prove that a canonical module for $C$ (if it exists) is unique up to isomorphism. Also, if $R$ has a canonical module, then every semidualizing module $C$ has a $C$-canonical module. We shall show that if a semidualizing module $C$ has a canonical module and belongs to $\mathcal{A}_{C}(R)$ (the Auslander class of $C$ ), then $R$ has a canonical module.

In Section 4, we discuss the case where $R$ is a Cohen-Macaulay ring. Let us denote the canonical module of a semidualizing module $C$ by $\omega_{C}$. As an application, we prove some new results concerning the existence of the canonical module over Cohen-Macaulay rings via $C$-canonical modules. For instance, Theorem 4.1 says that if the canonical module for a semidualizing $R$-module $C$ exists, then $\omega_{R}$ exists. More precisely:

Theorem: Let $R$ be a Cohen-Macaulay ring. The following are equivalent:

(i) $\omega_{R}$ exists;

(ii) $\omega_{C}$ exists for every semidualizing module $C$;

(iii) $\omega_{C}$ exists for some semidualizing module $C$.

It is known that a Cohen-Macaulay local ring is Gorenstein if and only if $R \cong \omega_{R}$. By the following result (which is Corollary 4.3), one can replace $R$ by an arbitrary 
semidualizing module.

Theorem: Let $R$ be a Cohen-Macaulay ring. The following are equivalent:

(i) $R$ is Gorenstein;

(ii) $\omega_{C} \cong C$ for every semidualizing module $C$;

(iii) $\omega_{C} \cong C$ for some semidualizing module $C$.

Sharp [17] showed that over a Cohen-Macaulay local ring with the canonical module $\omega_{R}$, any maximal Cohen-Macaulay $R$-module with finite injective dimension, is equal to a finite direct sum of copies of $\omega_{R}$ (see [17, Theorem $\left.2.1(\mathrm{v})\right]$ ). By the following result (Theorem 4.9), we obtain a similar representation for some subclasses of maximal Cohen-Macaulay $R$-modules, via $C$-canonical modules.

Theorem: Let $R$ be a Cohen-Macaulay ring, $C$ be a semidualizing module, and suppose $\omega_{C}$ exists. Let $M$ be a maximal Cohen-Macaulay $R$-module with $\mathcal{I}_{C^{-}}$ $\operatorname{id}(M)<\infty$. Then $M \cong \bigoplus_{i=1}^{t} \omega_{C}$ for some positive number $t$.

\section{Preliminaries}

Throughout this paper $R$ is a commutative Noetherian local ring with nonzero identity and $\operatorname{dim} R=n$. We denote the maximal ideal of $R$ by $\mathfrak{m}$, and the residue field $R / \mathfrak{m}$ by $k$. The minimal number of generators of a finitely generated $R$ module $M$ is denoted by $\mu(M)$, which is equal to $\operatorname{vdim}_{k}\left(M \otimes_{R} k\right)$. The type of a finitely generated $R$-module $M$ is denoted by $r_{R}(M)$ and is defined by $r_{R}(M)=$ $\operatorname{vdim}_{k} \operatorname{Ext}_{R}^{t}(k, M)$ where $t=\operatorname{depth}_{R} M$. In particular when $M$ is an Artinian $R$ module, one has $r(M)=\operatorname{vdim}_{k} \operatorname{Soc}(M)$, where $\operatorname{Soc}(M)=\left(0:_{M} \mathfrak{m}\right)$. The $\mathfrak{m}$-adic completion of $R$ is denoted by $\hat{R}$. We use $\mathrm{E}_{R}(k)$ to denote the injective hull of the residue field $k$. For each $i \in \mathbb{N} \bigcup\{0\}$, the $i$-th local cohomology module of $M$ with respect to the ideal $\mathfrak{a}$ is defined by

$$
\mathrm{H}_{\mathfrak{a}}^{i}(M)=\underset{n \in \mathbb{N}}{\lim _{\vec{N}}} \operatorname{Ext}_{R}^{i}\left(R / \mathfrak{a}^{n}, M\right)
$$

We refer the reader to [3] for more details about local cohomology modules.

Definition 2.1. A finitely generated $R$-module $C$ is called a semidualizing $R$ module if the homothety map $R \rightarrow \operatorname{Hom}_{R}(C, C)$ is an isomorphism, and $\operatorname{Ext}_{R}^{i}(C, C)$ $=0$ for all $i>0$.

For example, the ring itself is always a semidualizing $R$-module. Also, a dualizing module of a Cohen-Macaulay local ring is a semidualizing $R$-module. 
Definition 2.2. Let $C$ be a semidualizing module and $M$ be an $R$-module. Then $M$ is called $C$-injective if $M \cong \operatorname{Hom}_{R}(C, I)$ for some injective $R$-module $I$. The class of $C$-injective $R$-modules is denoted by $\mathcal{I}_{C}(R)$. Also, $M$ is called $C$-projective if $M \cong C \otimes_{R} P$ for some projective $R$-module $P$. The class of $C$-projective $R$ modules is denoted by $\mathcal{P}_{C}(R)$.

Consider the complex:

$$
X=0 \rightarrow M \rightarrow B^{0} \rightarrow B^{1} \rightarrow \cdots \rightarrow B^{n} \rightarrow \ldots
$$

where each $B^{i}$ is a $C$-injective $R$-module. This complex is called an augmented $\mathcal{I}_{C}$-injective resolution for $M$ whenever the following complex is exact:

$$
C \otimes_{R} X=0 \rightarrow C \otimes_{R} M \rightarrow C \otimes_{R} B^{0} \rightarrow C \otimes_{R} B^{1} \rightarrow \cdots \rightarrow C \otimes_{R} B^{n} \rightarrow \ldots
$$

Also, $\mathcal{I}_{C}$-injective dimension of $M\left(\right.$ or $\left.\mathcal{I}_{C}-\mathrm{id}(M)\right)$ is defined as:

$$
\mathcal{I}_{C}-\mathrm{id}(M):=\inf \left\{\sup \left\{n \geq 0 \mid X_{n} \neq 0\right\} X \text { is an } \mathcal{I}_{C} \text {-injective resolution of } M\right\} .
$$

The terms $\mathcal{P}_{C}$-projective resolution and $\mathcal{P}_{C}$-projective dimension of $M\left(\mathcal{P}_{C}-\operatorname{pd}(\mathrm{M})\right)$ are defined dually. These concepts are completely discussed in [18].

Definition 2.3. The Auslander class with respect to $C$, denoted by $\mathcal{A}_{C}(R)$, is a class of $R$-modules $M$ such that

(i) the natural map $M \rightarrow \operatorname{Hom}_{R}\left(C, C \otimes_{R} M\right)$ is an isomorphism and

(ii) $\operatorname{Tor}_{i}^{R}(C, M)=0=\operatorname{Ext}_{R}^{i}\left(C, C \otimes_{R} M\right)$ for all $i \geq 1$.

Definition 2.4. The Bass class with respect to $C$, denoted by $\mathcal{B}_{C}(R)$, is a class of $R$-modules $M$ such that

(i) the evaluation map $C \otimes_{R} \operatorname{Hom}_{R}(C, M) \rightarrow M$ is an isomorphism and

(ii) $\operatorname{Ext}_{R}^{i}(C, M)=0=\operatorname{Tor}_{i}^{R}\left(C, \operatorname{Hom}_{R}(C, M)\right)$ for all $i \geq 1$.

Next, we recall some known results concerning semidualizing $R$-modules which will be needed throughout this paper.

Proposition 2.5. Let $C$ be a semidualizing module.

(i) $C$ is a faithful $R$-module and therefore $\operatorname{Supp}_{R}(C)=\operatorname{Spec}(R)$ and $\operatorname{dim} C=$ $\operatorname{dim} R$. Also, $\operatorname{Ass}_{R}(R)=\operatorname{Ass}_{R}(C)$.

(ii) A sequence $\underline{x}$ of elements of $R$ is an $R$-sequence if and only if it is a $C$-sequence and in this situation $C / \underline{x} C$ is a semidualizing $R / \underline{x} R$-module. Moreover, $\operatorname{depth}_{R}(R)=\operatorname{depth}_{R}(C)$.

(iii) One has $\operatorname{Hom}_{R}(C, M) \neq 0$ for any nonzero $R$-module $M$. 
(iv) If $\varphi: R \rightarrow S$ is a flat ring homomorphism then $C \otimes_{R} S$ is a semidualizing $S$ module. The converse holds when $\varphi$ is a faithfully flat ring homomorphism.

Proof. For ( $i$ ) see [21, Proposition 2.1.16], for (ii) see [21, Theorem 2.2.6], for (iii) see [21, Corollary 2.1.17] and for (iv) see [21, Proposition 2.2.1].

Theorem 2.6. ([18, Theorem 2.11]) Let $C$ be a semidualizing $R$-module and let $M$ be an $R$-module.

(i) $\mathcal{I}_{C}-\operatorname{id}_{R}(M)=\operatorname{id}_{R}\left(C \otimes_{R} M\right)$ and $\operatorname{id}_{R}(M)=\mathcal{I}_{C}-\operatorname{id}_{R}\left(\operatorname{Hom}_{R}(C, M)\right)$.

(ii) $\mathcal{P}_{C}-\operatorname{pd}_{R}(M)=\operatorname{pd}_{R}\left(\operatorname{Hom}_{R}(C, M)\right)$ and $\operatorname{pd}_{R}(M)=\mathcal{P}_{C}-\operatorname{pd}_{R}\left(C \otimes_{R} M\right)$.

Theorem 2.7. Let $C$ be a semidualizing module.

(i) If any two $R$-modules in a short exact sequence are in $\mathcal{A}_{C}(R)$, respectively $\mathcal{B}_{C}(R)$, then so is the third.

(ii) $\mathcal{A}_{C}(R)$ (resp. $\mathcal{B}_{C}(R)$ ) contains every $R$-module of finite projective dimension (resp. injective dimension).

(iii) $\mathcal{A}_{C}(R)$ (resp. $\mathcal{B}_{C}(R)$ ) contains every $R$-module of finite $\mathcal{I}_{C}$-injective dimension (resp. $\mathcal{P}_{C}$-projective dimension).

Proof. (i) See [21, Proposition 3.1.7].

(ii) See [21, Proposition 3.1.9 and Proposition 3.1.10].

(iii) See [18, Corollary 2.9].

Theorem 2.8. ([18, Theorem 2.8]) Let $C$ be a semidualizing $R$-module and $M$ be an $R$-module.

(i) $M \in \mathcal{B}_{C}(R)$ if and only if $\operatorname{Hom}_{R}(C, M) \in \mathcal{A}_{C}(R)$.

(ii) $M \in \mathcal{A}_{C}(R)$ if and only if $C \otimes_{R} M \in \mathcal{B}_{C}(R)$.

\section{Main results}

In this section $C$ is a semidualizing $R$-module. We generalize some concepts which are stated in chapter 12 of [3].

Definition 3.1. A $C$-canonical module (or a canonical module for $C$ ) is a finitely generated $R$-module $K$ such that $\operatorname{Hom}_{R}\left(K, \mathrm{E}_{R}(R / \mathfrak{m})\right) \cong \mathrm{H}_{\mathfrak{m}}^{n}(C)$.

Example 3.2. If $D$ is the dualizing module for $R$, then $R$ is a $D$-canonical module.

Remark 3.3. If $R$ is a homomorphic image of an $n^{\prime}$-dimensional Gorenstein local ring $R^{\prime}$, then by the Local Duality Theorem ([3, Theorem 11.2.6]), $K=$ $\operatorname{Ext}_{R^{\prime}}^{n^{\prime}-n}\left(C, R^{\prime}\right)$ is a $C$-canonical module. 
Lemma 3.4. Let $R$ be a complete local ring. Then $C$ has a canonical module and any two $C$-canonical modules are isomorphic.

Proof. By Remark 3.3, and Cohen's Structure Theorem [14, Theorem 29.4], $C$ has a canonical module. If $K$ and $K^{\prime}$ are $C$-canonical modules, then

$$
\operatorname{Hom}_{R}\left(K, \mathrm{E}_{R}(R / \mathfrak{m})\right) \cong \operatorname{Hom}_{R}\left(K^{\prime}, \mathrm{E}_{R}(R / \mathfrak{m})\right) \text {. }
$$

Now $K \cong K^{\prime}$, by Matlis Duality Theorem [3, Theorem 10.2.12].

Theorem 3.5. Let $K$ be a finitely generated $R$-module. Then $K$ is a $C$-canonical module if and only if $K \otimes_{R} \hat{R}$ is a $C \otimes_{R} \hat{R}$-canonical module.

Proof. Note that $\mathrm{E}_{\hat{R}}(\hat{R} / \hat{\mathfrak{m}}) \cong \mathrm{E}_{R}(R / \mathfrak{m}) \otimes_{R} \hat{R}$ and by [3, Theorem 4.3.2], $\mathrm{H}_{\mathfrak{m}}^{n}(C) \otimes_{R} \hat{R} \cong \mathrm{H}_{\mathfrak{m}}^{n}\left(C \otimes_{R} \hat{R}\right)$. Let $K$ be a $C$-canonical module. Then by [14, Theorem 7.11], and [3, Theorem 4.3.2], there are $\hat{R}$-isomorphisms

$$
\begin{aligned}
\mathrm{H}_{\mathfrak{m}}^{n}\left(C \otimes_{R} \hat{R}\right) & \cong \mathrm{H}_{\mathfrak{m}}^{n}(C) \otimes_{R} \hat{R} \\
& \cong \operatorname{Hom}_{R}\left(K, \mathrm{E}_{R}(R / \mathfrak{m})\right) \otimes_{R} \hat{R} \\
& \cong \operatorname{Hom}_{\hat{R}}\left(K \otimes_{R} \hat{R}, \mathrm{E}_{R}(R / \mathfrak{m}) \otimes_{R} \hat{R}\right) \\
& \cong \operatorname{Hom}_{\hat{R}}\left(K \otimes_{R} \hat{R}, \mathrm{E}_{\hat{R}}(\hat{R} / \hat{\mathfrak{m}})\right) .
\end{aligned}
$$

Hence $K \otimes_{R} \hat{R}$ is a $C \otimes_{R} \hat{R}$-module.

Conversely, suppose that $K \otimes_{R} \hat{R}$ is a $C \otimes_{R} \hat{R}$-canonical $\hat{R}$-module. Therefore,

$$
\operatorname{Hom}_{\hat{R}}\left(K \otimes_{R} \hat{R}, \mathrm{E}_{\hat{R}}(\hat{R} / \hat{\mathfrak{m}})\right) \cong \mathrm{H}_{\mathfrak{m}}^{n}\left(C \otimes_{R} \hat{R}\right) .
$$

Using [14, Theorem 7.11], and [3, Theorem 4.3.2], we get

$$
\operatorname{Hom}_{R}\left(K, \mathrm{E}_{R}(R / \mathfrak{m})\right) \otimes_{R} \hat{R} \cong \mathrm{H}_{\mathfrak{m}}^{n}(C) \otimes_{R} \hat{R} .
$$

But both $R$-modules $\operatorname{Hom}_{R}\left(K, \mathrm{E}_{R}(R / \mathfrak{m})\right)$ and $\mathrm{H}_{\mathfrak{m}}^{n}(C)$ are Artinian, so that $\operatorname{Hom}_{R}\left(K, \mathrm{E}_{R}(R / \mathfrak{m})\right) \cong \mathrm{H}_{\mathfrak{m}}^{n}(C)($ See [3, Exercise 8.2.4]). Hence $K$ is a $C$-canonical module.

Theorem 3.6. Suppose that $K$ and $K^{\prime}$ are two $C$-canonical modules. Then $K \cong$ $K^{\prime}$.

Proof. By Theorem 3.5, $K \otimes_{R} \hat{R}$ and $K^{\prime} \otimes_{R} \hat{R}$ are $C \otimes_{R} \hat{R}$-canonical modules, so that by Lemma $3.4, K \otimes_{R} \hat{R} \cong K^{\prime} \otimes_{R} \hat{R}$. Now the result follows from [12, Lemma $5.8]$.

Remark 3.7. Suppose that there exists a $C$-canonical module. By Theorem 3.6, this $C$-canonical module is unique up to isomorphism. We shall denote this module by $\omega_{C}$. 
Theorem 3.8. (a) If $\omega_{R}$ exists, then $\operatorname{Hom}_{R}\left(C, \omega_{R}\right) \cong \omega_{C}$. Moreover, $\operatorname{Supp} \omega_{R}$ $=\operatorname{Supp} \omega_{C}$.

(b) Suppose that $\omega_{C}$ exists.

(i) $\operatorname{Ass}_{R} \omega_{C}=\{\mathfrak{p} \in \operatorname{Spec}(R): \operatorname{dim} R / \mathfrak{p}=n\}$.

(ii) Let $\operatorname{dim} R=n>0$, and let $a_{1}, \ldots a_{n}$ be a system of parameters for $R$. Then $a_{1}$ is a non-zerodivisor on $\omega_{C}$ and if $n \geq 2$, then $a_{1}, a_{2}$ is an $\omega_{C}$-sequence.

Proof. (a) By tensoring both sides of $\operatorname{Hom}_{R}\left(\omega_{R}, \mathrm{E}_{R}(R / \mathfrak{m})\right) \cong \mathrm{H}_{\mathfrak{m}}^{n}(R)$ with $C$, we get

$$
C \otimes_{R} \operatorname{Hom}_{R}\left(\omega_{R}, \mathrm{E}_{R}(R / \mathfrak{m})\right) \cong \mathrm{H}_{\mathfrak{m}}^{n}(R) \otimes_{R} C .
$$

But by [3, Lemma 10.2.16],

$$
C \otimes_{R} \operatorname{Hom}_{R}\left(\omega_{R}, \mathrm{E}_{R}(R / \mathfrak{m})\right) \cong \operatorname{Hom}_{R}\left(\operatorname{Hom}_{R}\left(C, \omega_{R}\right), \mathrm{E}_{R}(R / \mathfrak{m})\right)
$$

and $\mathrm{H}_{\mathfrak{m}}^{n}(R) \otimes_{R} C \cong \mathrm{H}_{\mathfrak{m}}^{n}(C)$ by [3, Exercise 6.1.10]. Next we show that $\operatorname{Supp} \omega_{R}=$ $\operatorname{Supp} \omega_{C}$. Let $\mathfrak{p} \in \operatorname{Supp} \omega_{R}$. By Proposition 2.5 (iv), $C_{\mathfrak{p}}$ is a semidualizing $R_{\mathfrak{p}^{-}}$ module and by Proposition 2.5 (iii), $\operatorname{Hom}_{R_{\mathfrak{p}}}\left(C_{\mathfrak{p}},\left(\omega_{R}\right)_{\mathfrak{p}}\right) \neq 0$. Hence $\left(\omega_{C}\right)_{\mathfrak{p}} \neq 0$ and thus $\mathfrak{p} \in \operatorname{Supp} \omega_{C}$. The converse inclusion holds because

$$
\operatorname{Supp} \omega_{C}=\operatorname{Supp}\left(\operatorname{Hom}_{R}\left(C, \omega_{R}\right)\right) \subseteq \operatorname{Supp} \omega_{R} .
$$

(b) The proof is similar to [3, Theorem 12.1.9].

The following result, shows that under special conditions, the existence of $\omega_{C}$ guarantees the existence of $\omega_{R}$.

Theorem 3.9. Suppose that $\omega_{C}$ exists. Then the following are equivalent:

(i) $\omega_{C} \in \mathcal{A}_{C}(R)$;

(ii) $\mathrm{H}_{\mathfrak{m}}^{n}(R) \in \mathcal{A}_{C}(R)$;

(iii) $\omega_{R}$ exists and belongs to $\mathcal{B}_{C}(R)$.

If the above equivalent conditions hold, then $\omega_{R} \cong C \otimes_{R} \omega_{C}$.

Proof. $(i) \Rightarrow(i i)$ One has

$$
\operatorname{Hom}_{R}\left(\omega_{C}, \mathrm{E}_{R}(R / \mathfrak{m})\right) \cong \mathrm{H}_{\mathfrak{m}}^{n}(C) \cong \mathrm{H}_{\mathfrak{m}}^{n}(R) \otimes_{R} C .
$$

Thus by hypothesis and [21, Proposition 3.3.1], $C \otimes_{R} \mathrm{H}_{\mathfrak{m}}^{n}(R) \in \mathcal{B}_{C}(R)$ and by Theorem 2.8, $\mathrm{H}_{\mathfrak{m}}^{n}(R) \in \mathcal{A}_{C}(R)$, as desired. 
$($ ii $) \Rightarrow($ iii $)$ Since $\mathrm{H}_{\mathfrak{m}}^{n}(R) \in \mathcal{A}_{C}(R)$, it follows that

$$
\mathrm{H}_{\mathfrak{m}}^{n}(R) \cong \operatorname{Hom}_{R}\left(C, C \otimes_{R} \mathrm{H}_{\mathfrak{m}}^{n}(R)\right) \cong \operatorname{Hom}_{R}\left(C, \mathrm{H}_{\mathfrak{m}}^{n}(C)\right) .
$$

Therefore, using Hom-Tensor adjointness and by $\operatorname{Hom}_{R}\left(\omega_{C}, \mathrm{E}_{R}(R / \mathfrak{m})\right) \cong \mathrm{H}_{\mathfrak{m}}^{n}(C)$, we can deduce that $\operatorname{Hom}_{R}\left(C \otimes_{R} \omega_{C}, \mathrm{E}_{R}(R / \mathfrak{m})\right) \cong \mathrm{H}_{\mathfrak{m}}^{n}(R)$, which shows that $\omega_{R}$ exists and is isomorphic to $C \otimes_{R} \omega_{C}$. Also, $\omega_{R} \in \mathcal{B}_{C}(R)$ holds by $\mathrm{H}_{\mathfrak{m}}^{n}(R) \in \mathcal{A}_{C}(R)$ and [21, Proposition 3.3.1].

$($ iii $) \Rightarrow(i)$ This is clear by Theorems 3.8 and 2.8 .

Let $u_{R}(0)$ be the intersection of the primary components $\mathfrak{q}$ of the zero ideal of $R$ for which $\operatorname{dim} R / \mathfrak{q}=n$.

Proposition 3.10. Let $S(C)$ denote the set of all submodules of $C$. Set

$$
\begin{gathered}
\Sigma:=\{N \in S(C): \operatorname{dim} N<n\} \\
=\left\{N \in S(C), N_{\mathfrak{p}}=0 \quad \text { for all } \mathfrak{p} \in \operatorname{Spec}(\mathrm{R}): \operatorname{dim} R / \mathfrak{p}=n\right\} .
\end{gathered}
$$

$B y\left[3\right.$, Lemma 7.3.1], $\Sigma$ has a largest element $N^{\prime}$. Then the following hold:

(i) $u_{R}(0)=\operatorname{Ann}_{R}\left(C / N^{\prime}\right)$;

(ii) If $\omega_{C}$ exists, then it is annihilated by $u_{R}(0)$;

(iii) Let $K$ be a finitely generated $R$-module which is annihilated by $u_{R}(0)$. Set $G:=C / N^{\prime}$ and $\bar{R}:=R / u_{R}(0)$ and $\overline{\mathfrak{m}}:=\mathfrak{m} / u_{R}(0)$. Then $K$ is a $C$ canonical module if and only if

$$
\operatorname{Hom}_{\bar{R}}\left(K, \mathrm{E}_{\bar{R}}(\bar{R} / \overline{\mathfrak{m}})\right) \cong \mathrm{H}_{\mathfrak{m}}^{n}(G)
$$

Proof. (i) If $\operatorname{dim} R / \mathfrak{p}=n$ then

$$
\operatorname{Ann}_{R}\left(C / N^{\prime}\right) R_{\mathfrak{p}}=\operatorname{Ann}_{R_{\mathfrak{p}}}\left(C_{\mathfrak{p}} / N_{\mathfrak{p}}^{\prime}\right)=\operatorname{Ann}_{R_{\mathfrak{p}}}\left(C_{\mathfrak{p}}\right)=0
$$

and the last equality holds because $C_{\mathfrak{p}}$ is a semidualizing $R_{\mathfrak{p}}$-module. Hence we have $\operatorname{dim}\left(\operatorname{Ann}_{R}\left(C / N^{\prime}\right)\right)<n$ and by [3, Exercise 12.1.11], $\operatorname{Ann}_{R}\left(C / N^{\prime}\right) \subseteq u_{R}(0)$. For the converse inclusion, let $r \in u_{R}(0)$. We show that $\operatorname{dim}(r C)<n$. Suppose that $\mathfrak{p} \in \operatorname{Spec}(R)$ such that $\operatorname{dim} R / \mathfrak{p}=n$. Note that $r R_{\mathfrak{p}}=0$ by [3, Exercise 12.1.11], and therefore $(r C)_{\mathfrak{p}}=r R_{\mathfrak{p}} C_{\mathfrak{p}}=0$. This shows that $\operatorname{dim}(r C)<n$. Since $N^{\prime}$ is the largest element of $\Sigma$, hence $r C \subseteq N^{\prime}$. Therefore, $r\left(C / N^{\prime}\right)=0$, and we have $u_{R}(0) \subseteq \operatorname{Ann}_{R}\left(C / N^{\prime}\right)$.

(ii) By [3, Lemma 7.3.1], we have $\mathrm{H}_{\mathfrak{m}}^{n}(C) \cong \mathrm{H}_{\mathfrak{m}}^{n}(G)$. But by part (i), $u_{R}(0)$ annihilates $G$, therefore it annihilates $\mathrm{H}_{\mathfrak{m}}^{n}(C)$. As $\operatorname{Hom}_{R}\left(\omega_{C}, \mathrm{E}_{R}(R / \mathfrak{m})\right) \cong \mathrm{H}_{\mathfrak{m}}^{n}(C)$, we conclude that $u_{R}(0)$ annihilates $\operatorname{Hom}_{R}\left(\omega_{C}, \mathrm{E}_{R}(R / \mathfrak{m})\right)$. By [3, Remark 10.2.2], 
$\operatorname{Hom}_{R}\left(\omega_{C}, \mathrm{E}_{R}(R / \mathfrak{m})\right)$ and $\omega_{C}$ have the same annihilator, therefore $u_{R}(0)$ annihilates $\omega_{C}$.

(iii) Let $K$ be a finitely generated $R$-module which is annihilated by $u_{R}(0)$. Using $\left(\left[3\right.\right.$, Theorem 4.2.1]), and $\mathrm{H}_{\mathfrak{m}}^{n}(C) \cong \mathrm{H}_{\mathfrak{m}}^{n}(G)$, we get $\mathrm{H}_{\mathfrak{m}}^{n}(C) \cong \mathrm{H}_{\mathfrak{m}}^{n}(G)$. Set $E:=\mathrm{E}_{R}(R / \mathfrak{m})$. In view of $[3$, Lemma 10.1.16], we have

$$
\begin{aligned}
\operatorname{Hom}_{R}(K, E) & \cong \operatorname{Hom}_{R}\left(K \otimes_{R} \bar{R}, E\right) \\
& \cong \operatorname{Hom}_{R}\left(K, \operatorname{Hom}_{R}(\bar{R}, E)\right) \\
& \cong \operatorname{Hom}_{R}\left(K,\left(0:_{E} u_{R}(0)\right)\right) \\
& \cong \operatorname{Hom}_{\bar{R}}\left(\left(K,\left(0:_{E} u_{R}(0)\right)\right)\right. \\
& \cong \operatorname{Hom}_{\bar{R}}\left(K, \mathrm{E}_{\bar{R}}(\bar{R} / \bar{m})\right) .
\end{aligned}
$$

Thus there is an $R$-isomorphism $\operatorname{Hom}_{R}(K, E) \cong \mathrm{H}_{\mathfrak{m}}^{n}(C)$ if and only if there is an $\bar{R}$-isomorphism $\operatorname{Hom}_{\bar{R}}\left(K, \mathrm{E}_{\bar{R}}(\bar{R} / \bar{m})\right) \cong \mathrm{H}_{\overline{\mathfrak{m}}}^{n}(G)$.

Theorem 3.11. Suppose that $\omega_{C}$ exists. Then $\left(0:_{R} \omega_{C}\right)=\left(0:_{R} H_{\mathfrak{m}}^{n}(C)\right)=u_{R}(0)$.

Proof. By [3, Remark 10.2.2], we have $\left(0:_{R} \omega_{C}\right)=\left(0:_{R} \mathrm{H}_{\mathfrak{m}}^{n}(C)\right)$. Note that $u_{R}(0) \subseteq\left(0:_{R} \omega_{C}\right)$ by Proposition 3.10 (ii). First suppose that $R$ is a homomorphic image of a Gorenstein local ring $\left(R^{\prime}, \mathfrak{m}^{\prime}\right)$ and therefore by [3, Remark 12.1.14], we may assume that $\operatorname{dim} R^{\prime}=n$. As $\omega_{R}$ exists, we have $\omega_{C} \cong \operatorname{Hom}_{R}\left(C, \omega_{R}\right)$. It is enough to show that $\left(0:_{R} \omega_{C}\right) R_{\mathfrak{p}}=0$ for all $\mathfrak{p} \in \operatorname{Spec}(R)$ with $\operatorname{dim} R / \mathfrak{p}=n$. But

$\left(0:_{R} \omega_{C}\right) R_{\mathfrak{p}}=\left(0:_{R_{\mathfrak{p}}}\left(\omega_{C}\right)_{\mathfrak{p}}\right)=\left(0:_{R_{\mathfrak{p}}} \operatorname{Hom}\left(C, \omega_{R}\right)_{\mathfrak{p}}\right)=\left(0:_{R_{\mathfrak{p}}} \operatorname{Hom}_{R_{\mathfrak{p}}}\left(C_{\mathfrak{p}},\left(\omega_{R}\right)_{\mathfrak{p}}\right)\right)$.

By $\left[3\right.$, Theorem 12.1 .18 (ii)], one has $\left(\omega_{R}\right)_{\mathfrak{p}} \cong \omega_{R_{\mathfrak{p}}}$. Note that $R_{\mathfrak{p}}$ is a 0 -dimensional local ring and $\omega_{R_{\mathfrak{p}}}$ is the canonical module for $R_{\mathfrak{p}}$, so that $\omega_{R_{\mathfrak{p}}} \cong \mathrm{E}_{R_{\mathfrak{p}}}\left(R_{\mathfrak{p}} / \mathfrak{p} R_{\mathfrak{p}}\right)$. Hence

$$
\left(0:_{R} \operatorname{Hom}_{R_{\mathfrak{p}}}\left(C_{\mathfrak{p}},\left(\omega_{R}\right)_{\mathfrak{p}}\right)\right)=\left(0:_{R_{\mathfrak{p}}} \operatorname{Hom}_{R_{\mathfrak{p}}}\left(C_{\mathfrak{p}}, \mathrm{E}_{R_{\mathfrak{p}}}\left(R_{\mathfrak{p}} / \mathfrak{p} R_{\mathfrak{p}}\right)\right)=\left(0:_{R_{\mathfrak{p}}} C_{\mathfrak{p}}\right)=0 .\right.
$$

We have proved the theorem when $R$ is a homomorphic image of a Gorenstein local ring. Now let $R$ be an arbitrary ring. An argument as in [3, Theorem 12.1.15], shows that $\left(0:_{R} \omega_{C}\right)=u_{R}(0)$.

Theorem 3.12. Suppose that $\omega_{C}$ exists.

(i) One has

$$
\operatorname{Supp} \omega_{C}=\{\mathfrak{p} \in \operatorname{Spec}(R): \operatorname{ht} \mathfrak{p}+\operatorname{dim} R / \mathfrak{p}=n\} ;
$$

moreover, $\omega_{C}$ and $\operatorname{Hom}_{R}\left(\omega_{C}, \omega_{C}\right)$ satisfy the condition $S_{2}$. 
(ii) If $R$ is a homomorphic image of a Gorenstein local ring, then $\left(\omega_{C}\right)_{\mathfrak{p}} \cong \omega_{C_{\mathfrak{p}}}$ for each $\mathfrak{p} \in \operatorname{Supp} \omega_{C}$.

Proof. First suppose that $R$ is a homomorphic image of a Gorenstein local ring $R^{\prime}$ with $\operatorname{dim} R^{\prime}=n$. Hence $\omega_{R}$ and $\omega_{C}$ exist, one has $\omega_{C} \cong \operatorname{Hom}_{R}\left(C, \omega_{R}\right)$, and $\operatorname{Supp} \omega_{R}=\operatorname{Supp} \omega_{C}$ by Theorem 3.8 (a). Now by [3, Theorem 12.1.18], the result follows. Next we prove that $\omega_{C}$ and $\operatorname{Hom}_{R}\left(\omega_{C}, \omega_{C}\right)$ are $S_{2} R$-modules. But let us first prove (ii). Let $\mathfrak{p} \in \operatorname{Supp} \omega_{C}$. By [3, Theorem 12.1.18], $\omega_{R \mathfrak{p}}$ exists and we have $\omega_{R \mathfrak{p}} \cong\left(\omega_{R}\right)_{\mathfrak{p}}$. Hence

$$
\left(\omega_{C}\right)_{\mathfrak{p}} \cong\left(\operatorname{Hom}_{R}\left(C, \omega_{R}\right)\right)_{\mathfrak{p}} \cong \operatorname{Hom}_{R_{\mathfrak{p}}}\left(C_{\mathfrak{p}},\left(\omega_{R}\right)_{\mathfrak{p}}\right) \cong \operatorname{Hom}_{R_{\mathfrak{p}}}\left(C_{\mathfrak{p}}, \omega_{R_{\mathfrak{p}}}\right) \cong \omega_{C_{\mathfrak{p}}} .
$$

It remains to show that $\omega_{C}$ and $\operatorname{Hom}_{R}\left(\omega_{C}, \omega_{C}\right)$ satisfy the condition $S_{2}$. Note that by Theorem $3.8(\mathrm{~b}), \operatorname{detph}_{R_{\mathfrak{p}}}\left(\omega_{C}\right)_{\mathfrak{p}} \geq \min \left\{2, \operatorname{dim} R_{\mathfrak{p}}\right\}$ as $\left(\omega_{C}\right)_{\mathfrak{p}}$ is a $C_{\mathfrak{p}}$-canonical module. Also, by [4, Exercise 1.4.19],

$$
\operatorname{depth}_{R_{\mathfrak{p}}}\left(\operatorname{Hom}_{R}\left(\left(\omega_{C}\right)_{\mathfrak{p}},\left(\omega_{C}\right)_{\mathfrak{p}}\right) \geq \min \left\{2, \operatorname{depth}_{R_{\mathfrak{p}}}\left(\omega_{C}\right)_{\mathfrak{p}}\right\} \geq \min \left\{2, \operatorname{dim} R_{\mathfrak{p}}\right\} .\right.
$$

Thus we have proved the theorem when $R$ is a homomorphic of a Gorenstein local ring. Now let $R$ be an arbitrary ring. Note that $\omega_{C \otimes_{R} \hat{R}} \cong \omega_{C} \otimes_{R} \hat{R}$ and $\hat{R}$ is a homomorphic image of a regular local ring, so that $\omega_{C} \otimes_{R} \hat{R}$ and $\operatorname{Hom}_{\hat{R}}\left(\omega_{C \otimes_{R} \hat{R}}, \omega_{C \otimes_{R} \hat{R}}\right)$ are $S_{2} \hat{R}$-modules. Let $\mathfrak{p} \in \operatorname{Spec}(R)$. There exists a prime ideal of $\hat{R}$ lying over $\mathfrak{p}$. Suppose that $\beta$ is the minimal ideal among these primes. We claim that $\beta$ is a minimal prime ideal of $\mathfrak{p} \hat{R}$. To see this, suppose that $\mathfrak{p} \hat{R} \subseteq \mathfrak{q} \subseteq \beta$ for some $\mathfrak{q} \in \operatorname{Spec}(\hat{R})$. Then

$$
\mathfrak{p} \subseteq(\mathfrak{p} \hat{R})^{c} \subseteq \mathfrak{q}^{c} \subseteq \beta^{c}=\mathfrak{p} .
$$

Now minimality of $\beta$ implies that $\beta=\mathfrak{q}$ as required. Thus $\operatorname{dim} \hat{R}_{\beta} / \mathfrak{p} \hat{R}_{\beta}=0$, so that by [14, Theorem 15.1], we have $\mathrm{ht}_{\hat{R}} \beta=\mathrm{ht}_{R} \mathfrak{p}$.

Consider the flat local ring homomorphism $R_{\mathfrak{p}} \longrightarrow \hat{R}_{\beta}$. By [14, Theorem 23.3],

$$
\operatorname{depth}_{\hat{R}_{\beta}}\left(\operatorname{Hom}\left(\left(\omega_{C}\right)_{\mathfrak{p}},\left(\omega_{C}\right)_{\mathfrak{p}}\right) \otimes_{R_{\mathfrak{p}}} \hat{R}_{\beta}\right)=\operatorname{depth}_{R_{\mathfrak{p}}} \operatorname{Hom}_{R_{\mathfrak{p}}}\left(\left(\omega_{C}\right)_{\mathfrak{p}},\left(\omega_{C}\right)_{\mathfrak{p}}\right) .
$$

$\operatorname{Thus~depth}_{R_{\mathfrak{p}}} \operatorname{Hom}_{R_{\mathfrak{p}}}\left(\left(\omega_{C}\right)_{\mathfrak{p}},\left(\omega_{C}\right)_{\mathfrak{p}}\right) \geq \min \left\{2, \mathrm{ht}_{\hat{R}} \beta\right\}=\min \left\{2, \mathrm{ht}_{R} \mathfrak{p}\right\}$. With a similar argument for $\left(\omega_{C}\right)_{\mathfrak{p}}$ we see that $\omega_{C}$ is $S_{2}$. For the rest, let $\mathfrak{p} \in \operatorname{Spec}(R)$, so that we can choose $\beta$ as above. Using the $\hat{R}_{\beta}$-isomorphism

$$
\left(\omega_{C} \otimes_{R} \hat{R}\right) \otimes_{\hat{R}} \hat{R}_{\beta} \cong\left(\omega_{C} \otimes_{R} R_{\mathfrak{p}}\right) \otimes_{R_{\mathfrak{p}}} \hat{R}_{\beta}
$$

we have $\mathfrak{p} \in \operatorname{Supp} \omega_{C}$ if and only if $\beta \in \operatorname{Supp}_{\hat{R}} \omega_{C} \otimes_{R} \hat{R}$. Now suppose that $\mathfrak{p} \in \operatorname{Supp} \omega_{C}$. Then $\beta \in \operatorname{Supp}_{\hat{R}} \omega_{C} \otimes_{R} \hat{R}$. Thus $\operatorname{ht}_{\hat{R}} \beta+\operatorname{dim}_{\hat{R}} \hat{R} / \beta=n$. But $\operatorname{dim} \hat{R} / \beta=\operatorname{dim} R / \mathfrak{p}$ and $\operatorname{ht}_{\hat{R}} \beta=\mathrm{ht}_{R} \mathfrak{p}$. Hence $\mathrm{ht}_{R} \mathfrak{p}+\operatorname{dim} R / \mathfrak{p}=n$ if and only if $\operatorname{ht}_{\hat{R}} \beta+\operatorname{dim}_{\hat{R}} \hat{R} / \beta=n$. Thus $\beta \in \operatorname{Supp} \omega_{C} \otimes_{R} \hat{R}$ and therefore $\mathfrak{p} \in \operatorname{Supp} \omega_{C}$. 
We end this section by asking two questions.

Question 3.13. Suppose that $\omega_{C}$ exists for some semidualizing module. Can we conclude that $\omega_{R}$ exists?

Question 3.14. Suppose that $\omega_{C} \cong \omega_{C^{\prime}}$ for two semidualizing modules $C$ and $C^{\prime}$. Does this imply that $C \cong C^{\prime}$ ?

These questions will be answered when $R$ is a Cohen-Macaulay ring (see Theorem 4.1, and Corollary 4.5 (ii)).

\section{The Cohen-Macaulay case}

In this section, we present some results concerning $C$-canonical $R$-modules, when $R$ is a Cohen-Macaulay local ring and $C$ is a semidualizing module. By [3, Corollary 12.1.21], we know that if $\omega_{R}$ exists then it has finite injective dimension and $\omega_{R}$ is the dualizing module.

Theorem 4.1. Let $R$ be a Cohen-Macaulay ring. The following are equivalent:

(i) $\omega_{R}$ exists;

(ii) $\omega_{C}$ exists for every semidualizing module $C$;

(iii) $\omega_{C}$ exists for some semidualizing module $C$.

Proof. $(i) \Rightarrow(i i)$ This is clear by Theorem 3.8 (a).

(ii) $\Rightarrow$ (iii) It is obvious.

(iii) $\Rightarrow\left(\right.$ i) We claim that $C \otimes_{R} \omega_{C} \cong \omega_{R}$. In view of Theorem 3.5, it is enough for us to show that $\left(C \otimes_{R} \omega_{C}\right) \otimes_{R} \hat{R} \cong \omega_{\hat{R}}$. But $\left(C \otimes_{R} \omega_{C}\right) \otimes_{R} \hat{R} \cong\left(C \otimes_{R} \hat{R}\right) \otimes_{\hat{R}}\left(\omega_{C} \otimes_{R} \hat{R}\right)$ and by Theorem 3.5, $\omega_{C} \otimes_{R} \hat{R}$ is a canonical module for $\hat{R}$-semidualizing module $C \otimes_{R} \hat{R}$. Thus we assume that $R$ is complete, and therefore $\omega_{R}$ exists. Hence by Theorem $3.8(\mathrm{a}), \omega_{C} \cong \operatorname{Hom}_{R}\left(C, \omega_{R}\right)$. Note that $R$ is Cohen-Macaulay and therefore $\operatorname{id}_{R}\left(\omega_{R}\right)<\infty$. It follows from Theorem 2.7 that $\omega_{R} \in \mathcal{B}_{C}(R)$, and therefore $C \otimes_{R} \omega_{C} \cong C \otimes_{R} \operatorname{Hom}_{R}\left(C, \omega_{R}\right) \cong \omega_{R}$.

Corollary 4.2. Let $R$ be a Cohen-Macaulay ring and suppose $\omega_{C}$ exists. Then $\mathcal{I}_{C}-\mathrm{id} \omega_{C}<\infty$.

Proof. By Theorem 4.1, and Theorem 3.8 (a), $\omega_{R}$ exists and $\omega_{C}$ is isomorphic to $\operatorname{Hom}_{R}\left(C, \omega_{R}\right)$. Now Theorem 2.6 implies that $\mathcal{I}_{C}$-id $\omega_{C}=\operatorname{id}{ }_{R} \omega_{R}=n<\infty$.

Corollary 4.3. Let $R$ be a Cohen-Macaulay ring. The following are equivalent:

(i) $R$ is Gorenstein;

(ii) $\omega_{C} \cong C$ for every semidualizing module $C$; 
(iii) $\omega_{C} \cong C$ for some semidualizing module $C$.

Proof. $(i) \Rightarrow($ ii $)$ By [21, Corollary 4.1.11], the only semidualizing module over a Gorenstein local ring is $R$ itself and so (ii) holds.

(ii) $\Rightarrow$ (iii) Is clear.

(iii) $\Rightarrow\left(\right.$ i) By Corollary 4.2, we have $\mathcal{I}_{C}$-id $C<\infty$ and therefore $C \in \mathcal{A}_{C}(R)$ by Theorem 2.7. Hence $C$ is projective by [21, Corollary 4.3.2]. As $R$ is a local ring and $C$ is an indecomposable $R$-module, $C$ is a free $R$-module of rank 1 which shows that $C \cong R$. Therefore, $\operatorname{id}_{R} R<\infty$.

Remark 4.4. Let $C$ be a semidualizing $R$-module. If $R$ is a Cohen-Macaulay ring and $\omega_{C}$ exists, then by Theorem $4.1, \omega_{R}$ exsits and $\omega_{C} \cong \operatorname{Hom}_{R}\left(C, \omega_{R}\right)$. In this situation, by [21, Corollary 4.1.3], $\omega_{C}$ is a semidualizing $R$-module.

If $R$ is a Cohen-Macaulay local ring with the dualizing module $D$, then $D \cong \omega_{R}$ and $R \cong \omega_{D}$. Next we generalize this fact for any two semidualizing $R$-modules.

Corollary 4.5. Let $R$ be a Cohen-Macaulay ring and $C$ and $C^{\prime}$ be two semidualizing modules.

(i) One has $\omega_{C} \cong C^{\prime}$ if and only if $\omega_{C^{\prime}} \cong C$.

(ii) If $\omega_{C} \cong \omega_{C^{\prime}}$, then $C \cong C^{\prime}$.

Proof. (i) Suppose that $\omega_{C} \cong C^{\prime}$. Then $\operatorname{Hom}_{R}\left(C, \omega_{R}\right) \cong C^{\prime}$. Since $C$ is a maximal Cohen-Macaulay $R$-module, hence by [4, Theorem 3.3.10], we have

$$
\begin{aligned}
C & \cong \operatorname{Hom}_{R}\left(\operatorname{Hom}_{R}\left(C, \omega_{R}\right), \omega_{R}\right) \\
& \cong \operatorname{Hom}_{R}\left(C^{\prime}, \omega_{R}\right) \cong \omega_{C^{\prime}} .
\end{aligned}
$$

(ii) The proof is similar to $(i)$.

Using Theorem 4.1, and [4, Theorem 3.3.10 and Proposition 3.3.11], one has the following result.

Proposition 4.6. Let $R$ be a Cohen-Macaulay ring and suppose that $\omega_{C}$ exists. Then $\mu\left(\omega_{C}\right)=r(C)$ and $r\left(\omega_{C}\right)=\mu(C)$.

Lemma 4.7. Let $R$ be a Cohen-Macaulay ring and suppose that $\omega_{C}$ exists. Then any $R$-sequence $\underline{x}$ is an $\omega_{C}$-sequence and we have $\omega_{C} / \underline{x} \omega_{C} \cong \omega_{C / \underline{x} C}$.

Proof. By Remark $4.4, \omega_{C}$ is a semidualizing module. Hence any $R$-sequence is an $\omega_{C}$-sequence by Proposition $2.5(i i)$. For the rest, note that $\omega_{C} \cong \operatorname{Hom}_{R}\left(C, \omega_{R}\right)$. Therefore,

$$
\omega_{C} \otimes_{R} R / \underline{x} \cong \omega_{C} / \underline{x} \omega_{C} \cong R / \underline{x} R \otimes_{R} \operatorname{Hom}_{R}\left(C, \omega_{R}\right) .
$$


By [4, Proposition 3.3.3], the right-hand side is isomorphic to

$\operatorname{Hom}_{R / \underline{x} R}\left(C / \underline{x} C, \omega_{R} / \underline{x} \omega_{R}\right)$ and this is isomorphic to $\omega_{C / \underline{x} C}$.

Theorem 4.8. Let $R$ be a Cohen-Macaulay ring and suppose that $\omega_{C}$ exists. Let $N$ be a maximal Cohen-Macaulay $R$-module in $\mathcal{A}_{C}(R)$. Then:

(i) $\operatorname{Hom}_{R}\left(N, \omega_{C}\right)$ is a maximal Cohen-Macaulay $R$-module in $\mathcal{A}_{C}(R)$.

(ii) $N \cong \operatorname{Hom}_{R}\left(\operatorname{Hom}_{R}\left(N, \omega_{C}\right), \omega_{C}\right)$ (that is, $N$ is $\omega_{C}$-reflexive).

(iii) For any $R$-sequence $\underline{x}$ one has

$$
\operatorname{Hom}_{R}\left(N, \omega_{C}\right) \otimes_{R} R / \underline{x} R \cong \operatorname{Hom}_{R / \underline{x} R}\left(N / \underline{x} N, \omega_{C} / \underline{x} \omega_{C}\right) .
$$

Proof. (i) By Theorem 4.1, $\omega_{R}$ exists. Also, $\operatorname{Ext}_{\mathrm{R}}^{\mathrm{j}}\left(\mathrm{N}, \omega_{\mathrm{R}}\right)=0$ for all $j>0$ and $\operatorname{Hom}_{R}\left(N, \omega_{R}\right)$ is a maximal Cohen-Macaulay $R$-module by [4, Theorem 3.3.10]. Thus by [21, Proposition 3.3.16], we have $\operatorname{Hom}_{R}\left(N, \omega_{R}\right) \in \mathcal{B}_{C}(R)$. Since $\operatorname{Ext}_{R}^{j}\left(C, \operatorname{Hom}_{R}\left(N, \omega_{R}\right)\right)=0$ for all $j>0$, so that by [4, Proposition 3.3.3], $\operatorname{Hom}_{R}\left(C, \operatorname{Hom}_{R}\left(N, \omega_{R}\right)\right)$ is a maximal Cohen-Macaulay $R$-module and by Theorem 2.8 , it belongs to $\mathcal{A}_{C}(R)$. But this module is isomorphic to $\operatorname{Hom}_{R}\left(N, \operatorname{Hom}_{R}\left(C, \omega_{R}\right)\right)$ $\cong \operatorname{Hom}_{R}\left(N, \omega_{C}\right)$. This completes the proof of (i).

(ii) Using $\omega_{C} \cong \operatorname{Hom}_{R}\left(C, \omega_{R}\right)$ and Hom-Tensor adjointness, one has

$$
\begin{gathered}
\operatorname{Hom}_{R}\left(\operatorname{Hom}_{R}\left(N, \omega_{C}\right), \omega_{C}\right) \cong \operatorname{Hom}_{R}\left(C, \operatorname{Hom}_{R}\left(\operatorname{Hom}_{R}\left(N, \omega_{C}\right), \omega_{R}\right)\right) \\
\cong \operatorname{Hom}_{R}\left(C, \operatorname{Hom}_{R}\left(\operatorname{Hom}_{R}\left(C \otimes_{R} N, \omega_{R}\right), \omega_{R}\right)\right) .
\end{gathered}
$$

By [7, Lemma 2.11], $N \otimes_{R} C$ is a maximal Cohen-Macaulay $R$-module, so that by $[4$, Theorem 3.3.10 (d)],

$$
C \otimes_{R} N \cong \operatorname{Hom}_{R}\left(\operatorname{Hom}_{R}\left(C \otimes_{R} N, \omega_{R}\right), \omega_{R}\right) .
$$

Hence

$$
\operatorname{Hom}_{R}\left(\operatorname{Hom}_{R}\left(N, \omega_{C}\right), \omega_{C}\right) \cong \operatorname{Hom}_{R}\left(C, C \otimes_{R} N\right) .
$$

As $N \in \mathcal{A}_{C}(R)$, the right-hand side is isomorphic to $N$, as desired.

(iii) By [4, Proposition 3.3.3], and part (i), the following isomorphism holds:

$$
\begin{gathered}
\operatorname{Hom}_{R}\left(C, \operatorname{Hom}_{R}\left(N, \omega_{R}\right)\right) \otimes_{R} R / \underline{x} R \cong \\
\operatorname{Hom}_{R / \underline{x} R}\left(C / \underline{x} C, \operatorname{Hom}_{R}\left(N, \omega_{R}\right) / \underline{x} \operatorname{Hom}_{R}\left(N, \omega_{R}\right)\right) .
\end{gathered}
$$

The left-hand side is isomorphic to $\operatorname{Hom}_{R}\left(N, \omega_{C}\right) \otimes_{R} R / \underline{x} R$. Again by [4, Proposition 3.3.3], we conclude that the right-hand side is isomorphic to

$$
\operatorname{Hom}_{R / \underline{x} R}\left(C / \underline{x} C, \operatorname{Hom}_{R / \underline{x} R}\left(N / \underline{x} N, \omega_{R} / \underline{x} \omega_{R}\right)\right)
$$


and by using Hom-Tensor adjointness, the later is isomorphic to

$$
\operatorname{Hom}_{R / \underline{x} R}\left(N / \underline{x} N, \operatorname{Hom}_{R / \underline{x} R}\left(C / \underline{x} C, \omega_{R} / \underline{x} \omega_{R}\right)\right) \cong \operatorname{Hom}_{R / \underline{x} R}\left(N / \underline{x} N, \omega_{C} / \underline{x} \omega_{C}\right)
$$

where the last isomorphism is obtained from Lemma 4.7.

Theorem 4.9. Let $R$ be a Cohen-Macaulay ring and suppose that $\omega_{C}$ exists and $M$ is a maximal Cohen-Macaulay $R$-module with $\mathcal{I}_{C}-\mathrm{id}(M)<\infty$. Then $M \cong \bigoplus_{i=1}^{t} \omega_{C}$ for some positive number $t$.

Proof. By Theorem 2.6, we have $\operatorname{id}_{R}\left(C \otimes_{R} M\right)<\infty$ and $M \in \mathcal{A}_{C}(R)$ by Theorem 2.7. Also, $C \otimes_{R} M$ is a maximal Cohen-Macaulay $R$-module by [7, Lemma 2.11]. Hence by $\left[17\right.$, Theorem 2.1(v)], we get $C \otimes_{R} M \cong \bigoplus_{i=1}^{t} \omega_{R}$. Applying $\operatorname{Hom}_{R}(C,-)$ on both sides, we get

$$
\operatorname{Hom}_{R}\left(C, C \otimes_{R} M\right) \cong \bigoplus_{i=1}^{t} \operatorname{Hom}_{R}\left(C, \omega_{R}\right) \cong \bigoplus_{i=1}^{t} \omega_{C} .
$$

But the left-hand side is isomorphic to $M$ because $M \in \mathcal{A}_{C}(R)$.

Remark 4.10. According to [4, Definition 3.3.1], the canonical module over a Cohen-Macaulay local ring, is a maximal Cohen-Macaulay R-module $K$ with finite injective dimension of type 1 . By the next theorem, one may define a $C$-canonical module as a maximal Cohen-Macaulay $R$-module with $r_{R}(K)=\mu(C)$ and $\mathcal{I}_{C^{-}}$ $\operatorname{id}(K)<\infty$.

Theorem 4.11. Let $R$ be a Cohen-Macaulay ring and $K$ be a finitely generated $R$-module. Then $K$ is a $C$-canonical module if and only if $K$ is a maximal CohenMacaulay module with $r_{R}(K)=\mu(C)$ and $\mathcal{I}_{C}$-id $(K)<\infty$.

Proof. $(\Rightarrow)$ This is clear by Corollary 4.2, Remark 4.4, and Proposition 4.6. $(\Leftarrow)$ Note that $K \otimes_{R} \hat{R}$ is a maximal Cohen-Macaulay $\hat{R}$-module and

$$
\mu(C)=r_{R}(K)=r_{\hat{R}}\left(K \otimes_{R} \hat{R}\right)=\mu\left(C \otimes_{R} \hat{R}\right) .
$$

By using Theorem 2.6, the following equalities hold:

$$
\begin{aligned}
\mathcal{I}_{C \otimes_{R} \hat{R}^{-i d}\left(K \otimes_{R} \hat{R}\right)} & =\operatorname{id}_{\hat{R}}\left(\left(K \otimes_{R} \hat{R}\right) \otimes_{\hat{R}}\left(C \otimes_{R} \hat{R}\right)\right) \\
& =\operatorname{id}_{\hat{R}}\left(\left(K \otimes_{R} C\right) \otimes_{R} \hat{R}\right) \\
& =\operatorname{id}_{R}\left(K \otimes_{R} C\right) \\
& =\mathcal{I}_{C}-\mathrm{id}(K)<\infty .
\end{aligned}
$$

Hence $I_{C \otimes_{R} \hat{R}}-\operatorname{id}\left(K \otimes_{R} \hat{R}\right)<\infty$. As $\omega_{C \otimes_{R} \hat{R}}$ exists, it is enough for us to show that $K \otimes_{R} \hat{R} \cong \omega_{C \otimes_{R} \hat{R}}$ by Theorem 3.5. Thus we may assume that $R$ is complete and 
therefore $\omega_{C}$ exists. By Theorem $4.9, K \cong \bigoplus_{i=1}^{t} \omega_{C}$ for some positive number $t$, so that $r_{R}(K)=\operatorname{tr}\left(\omega_{C}\right)=t \mu(C)$. But $r_{R}(K)=\mu(C)=r\left(\omega_{C}\right)$ which implies that $t=1$ and therefore $K \cong \omega_{C}$.

The following result is a generalization of [4, Proposition 3.3.13].

Theorem 4.12. Let $R$ be a Cohen-Macaulay ring and $C$ be a semidualizing $R$ module. For any $R$-module $Q$, the following conditions are equivalent.

(i) $Q \cong \omega_{C}$.

(ii) $Q \in \mathcal{A}_{C}(R)$ is a maximal Cohen-Macaulay faithful $R$-module and $r_{R}(Q)=$ $\mu(C)$.

Proof. $(i) \Rightarrow(i i)$. By remark $4.4, \omega_{C}$ is a semidualizing $R$-module. Therefore, it is a maximal Cohen-Macaulay faithful $R$-module. Also, by Proposition 4.6, $r\left(\omega_{C}\right)=$ $\mu(C)$.

(ii) $\Rightarrow($ i $)$. We claim that $C \otimes_{R} Q \cong \omega_{R}$. One can use [21, Proposition 3.4.7], and the exact sequence $0 \rightarrow R \stackrel{x}{\rightarrow} R \rightarrow R / x R \rightarrow 0$ to see that $Q / x Q \in \mathcal{A}_{C / x C}(R / x R)$ for any non-zero divisor $x$, and so that for any $R$-sequence $\underline{x}, Q / \underline{x} Q \in \mathcal{A}_{C / \underline{x} C}(R / \underline{x} R)$. Note that $C \otimes_{R} Q$ is a maximal Cohen-Macaulay $R$-module by [7, Lemma 2.11]. First we prove that $r_{R}\left(C \otimes_{R} Q\right)=1$. Let $\underline{x}$ be a maximal $R$-sequence. Note that $r_{R}(Q)=v \operatorname{dim}_{k} \operatorname{Ext}_{R}^{n}(k, Q)$ and the later is equal to $v \operatorname{dim}_{k} \operatorname{Hom}_{R / \underline{x}}(k, Q / \underline{x} Q)$. Set $\bar{R}:=R / \underline{x}, \bar{Q}:=Q / \underline{x} Q, \bar{C}:=C / \underline{x} C$ and $\overline{C \otimes_{R} Q}:=\left(C \otimes_{R} Q\right) / \underline{x}\left(C \otimes_{R} Q\right)$. By using Hom-Tensor adjointness, [4, Lemma 3.1.16], and $\bar{Q} \in A_{\bar{C}}(\bar{R})$, we have the following equalities:

$$
\begin{aligned}
r_{R}(Q) & =v \operatorname{dim}_{k} \operatorname{Hom}_{\bar{R}}\left(k, \operatorname{Hom}_{\bar{R}}\left(\bar{C}, \bar{C} \otimes_{\bar{R}} \bar{Q}\right)\right) \\
& =v \operatorname{dim}_{k} \operatorname{Hom}_{\bar{R}}\left(k, \operatorname{Hom}_{\bar{R}}\left(\bar{C}, \bar{R} \otimes_{R}\left(C \otimes_{R} Q\right)\right)\right. \\
& =v \operatorname{dim}_{k} \operatorname{Hom}_{\bar{R}}\left(k \otimes_{\bar{R}} \bar{C}, \overline{C \otimes_{R} Q}\right) \\
& =v \operatorname{dim}_{k} \operatorname{Hom}_{\bar{R}}\left(\bar{C}, \operatorname{Hom}_{\bar{R}}\left(k, \overline{C \otimes_{R} Q}\right)\right) \\
& =\mu_{\bar{R}}(\bar{C}) \cdot r_{\bar{R}}\left(\overline{C \otimes_{R} Q}\right) \\
& =\mu_{R}(C) \cdot r_{R}\left(C \otimes_{R} R\right) .
\end{aligned}
$$

By hypothesis $r_{R}(Q)=\mu(C)$, thus $r_{R}\left(C \otimes_{R} Q\right)=1$. On the other hand $Q \cong$ $\operatorname{Hom}_{R}\left(C, C \otimes_{R} Q\right)$. As $\mathrm{Q}$ is a faithful R-module we get $C \otimes_{R} Q$ is faithful. Hence by [4, Proposition 3.3.13], $\omega_{R}$ exists and is isomorphic to $C \otimes_{R} Q$. Therefore,

$$
Q \cong \operatorname{Hom}_{R}\left(C, C \otimes_{R} Q\right) \cong \operatorname{Hom}_{R}\left(C, \omega_{R}\right) \cong \omega_{C} .
$$


By using Theorem 4.12 and Corollary 4.5, we have the following result.

Corollary 4.13. Let $R$ be a Cohen-Macaulay ring. If $r_{R}(R)=\mu(C)$, then $C$ is the canonical module of $R$.

Acknowledgment. The authors would like to thank the referee for the valuable suggestions and comments.

\section{References}

[1] Y. Aoyama, On the depth and the projective dimension of the canonical module, Japan. J. Math., 6 (1980), 61-66.

[2] Y. Aoyama, Some basic results on canonical modules, J. Math. Kyoto Univ., 23 (1983), 85-94.

[3] M. P. Brodmann, R. Y. Sharp, Local Cohomology: An Algebraic Introduction with Geometric Applications, Second edition, Cambridge University Press, 2013.

[4] W. Bruns and J. Herzog, Cohen-Macaulay Rings, Revised edition, Cambridge University Press, Cambridge, 1993.

[5] L. W. Christensen, Semi-dualizing complexes and their Auslander categories, Trans. Amer. Math. Soc., 353(5) (2001), 1839-1883.

[6] L. W. Christensen and S. Sather-Wagstaff, A Cohen-Macaulay algebra has only finitely many semidualizing modules, Math. Proc. Cambridge Philos. Soc., 145(3) (2008), 601-603.

[7] Mohammad T. Dibaei and Arash Sadeghi, Linkage of modules and the Serre conditions, J. Pure Appl. Algebra, 219 (2015), 4458-4478.

[8] H.-B. Foxby, Gorenstein modules and related modules, Math. Scand., 31 (1972), 267-284.

[9] A. J. Frankild, S. Sather-Wagstaff, and A. Taylor, Relations between semidualizing complexes, J. Commut. Algebra, 1(3) (2009), 393-436.

[10] E. S. Golod, G-dimension and generalized perfect ideals, Trudy Mat. Inst. Steklov., 165 (1984), 62-66.

[11] R. Hartshorne, Local Cohomology, A seminar given by A. Grothendieck, Harvard University, Fall 1961, Springer-Verlag, Berlin-New York, 1967.

[12] J. Herzog and E. Kunz, Der kanonische Modul eines Cohen-Macaulay-Rings, Lect. Notes in Math., Vol. 238, Springer-Verlag, Berlin-New York, 1971.

[13] M. Hochster and C. Huneke, Indecomposable canonical modules and connectedness, Commutative algebra: syzygies, multiplicities, and birational algebra 
(South Hadley, MA, 1992), Contemp. Math., vol. 159, Amer. Math. Soc., Providence, RI, 1994, pp. 197-208.

[14] H. Matsumura, Commutative Ring Theory, Cambridge Studies in Advanced Mathematics, Vol. 8, Cambridge University Press, Cambridge, 1986.

[15] S. Nasseh and S. Sather-Wagstaff, Geometric aspects of representation theory for DG algebras: answering a question of Vasconcelos, J. Lond. Math. Soc. (2), 96(1) (2017), 271-292.

[16] I. Reiten, The converse to a theorem of Sharp on Gorenstein modules, Proc. Amer. Math. Soc., 32 (1972), 417-420.

[17] R. Y. Sharp, Finitely generated modules of finite injective dimension over certain Cohen-Macaulay rings, Proc. Lond. Math. Soc. (3), 25 (1972), 303-328.

[18] R. Takahashi and D. White, Homological aspects of semidualizing modules, Math. Scand., 106(1) (2010), 5-22.

[19] W. V. Vasconcelos, Divisor Theory in Module Categories, North-Holland Mathematics Studies, No. 14., North-Holland Publishing Co., AmsterdamOxford, 1974.

[20] S. Sather-Wagstaff, Semidualizing modules and the divisor class group, Illinois J. Math., 51(1) (2007), 255-285.

[21] S. Sather-Wagstaff, Semidualizing Modules, in preperation, URL: https://www.ndsu.edu/pubweb/ ssatherw/DOCS/sdm.pdf.

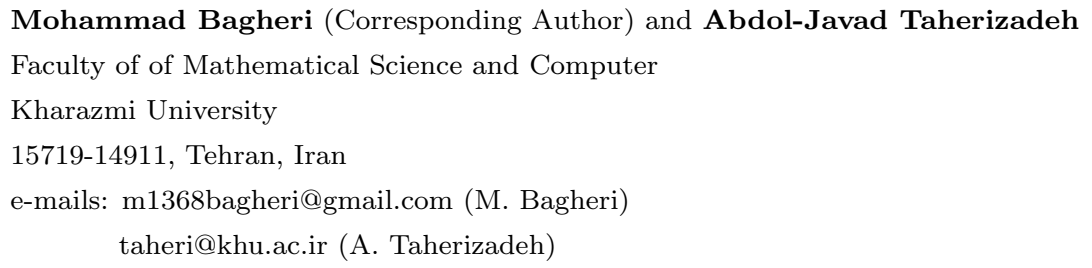

